\title{
PANDAS tras amigdalectomía
}

\section{PANDAS following amygdalectomy}

S. Aróstegui ${ }^{1}$, J.A. Agüero' ${ }^{2}$ C. Éscar ${ }^{2}$

\section{RESUMEN}

Se denomina PANDAS (Pediatric Autoinmune Neuropsychiatric Disorders Associated with Streptococcal Infections) al trastorno que se presenta en un subgrupo de pacientes con síntomas obsesivos y/o tics que aparecen relacionados con infecciones por estreptococo beta- hemolítico del grupo $\mathrm{A}$.

El caso clínico que se presenta corresponde a una niña de 5 años que desarrolla un trastorno obsesivocompulsivo en relación temporal con una amigdalectomía. Dicha paciente cumple los criterios diagnósticos y la evolución longitudinal correspondiente a este tipo de trastornos.

Palabras clave. PANDAS. Trastorno obsesivo-compulsivo. Amigdalectomía.

\section{ABSTRACT}

PANDAS (Pediatric Autoimmune Neuropsychiatric Disorders Associated with Streptococcal Infections) is the denomination for the disorder shown by a subgroup of patients with obsessive symptoms and/or subgroup of patients with obsessive symptoms and/or
tics that appear in relation to infections by group A tics that appear in relation
beta-hemolytic streptococci.

The clinical case that we present corresponds to a girl of 5 years who developed a compulsive-obsessive disorder in temporal relation to an amygdalectomy. This patient fulfilled the diagnostic criteria and the longitudinal evolution corresponding to this type of disorder.

Key words. PANDAS. Obsessive-compulsive disorder. Amygdalectomy.

An. sis. sanit. Navar. 2003; 26 (2): 287-290.

1. Unidad de Hospitalización Psiquiátrica. Hospital de Navarra.

2. Centro de Salud Mental. Tudela.

Aceptado para su publicación el 19 de marzo de 2003
Correspondencia

Silvia Aróstegui Uranga

Unidad de Hospitalización Psiquiátrica II (Pabellón E)

Hospital de Navarra

C/ Irunlarrea, 3

31008 Pamplona

Tfno: 948422254 


\section{INTRODUCCIÓN}

La aparición de síntomas obsesivos en diferentes trastornos neurológicos ${ }^{1}$ es un hecho bien conocido. Esta sintomatología es especialmente frecuente en los casos de fiebre reumática y sobre todo en la corea de Sydenham². En los últimos años se está estudiando la asociación temporal existente entre la aparición de clínica obsesiva $\mathrm{y} / \mathrm{o}$ tics en niños con infecciones por estreptococo b-hemolítico del grupo A. Se ha denominado a este trastorno con el acrónimo PANDAS (Pediatric Autoinmune Neuropsychiatric Disorders Associated with Streptococcal Infections).

Los primeros casos descritos de PANDAS han mostrado que aunque presentan los síntomas típicos del trastorno obsesivo-compulsivo (TOC) o de los trastornos por tics, aparecen algunas características diferenciales. Por este motivo se han propuesto los criterios diagnósticos de PAN$\mathrm{DAS}^{3}$ (Tabla 1).

Presentamos, según la bibliografía consultada, el primer caso de PANDAS detectado en Navarra y el primero descrito en la literatura en España, que presenta además la peculiaridad de haberse desencadenado tras una amigdalectomía.

\section{CASO CLÍNICO}

Se trata de una niña de 5 años que no presentó alteraciones durante el embarazo, parto o neurodesarrollo. Como antecedentes psiquiátricos familiares presenta al abuelo materno diagnosticado de trastorno límite de la personalidad y a la madre diagnosticada de TOC. Existen múltiples antecedentes médicos en la niña: crisis vasovagales, otitis serosa de repetición, adenoidectomía, infecciones urinarias repetidas sin anomalías en aparato urinario, dolores inespecíficos y amigdalitis de repetición.

Por este último motivo se le practica una amigdalectomía en febrero de 2002. A los 8 días de esta intervención, la niña comienza a presentar de forma súbita (la clínica aparece al levantarse, después de haberse acostado asintomática) un cuadro caracterizado fundamentalmente por ideas obsesivas. El contenido principal de estas ideas consistía en miedo a haberse comido cosas (animales, sus heces, personas, objetos inanimados...), repitiendo de forma cons tante preguntas acerca de este tema (por ejemplo: "mama, ¿verdad que no me he comido a esa señora?", “me he comido esa farola?”). Presentaba también temores de ser contaminada por otros niños y existían asociados trastornos de conducta (p.e.: pataletas, conductas oposicionistas), sintomatología ansiosa y ligera hiperactividad motora.

Fue remitida por su pediatra al Centro de Salud Mental (CSM), donde el psicólogo inició tratamiento conductual. Ante la persistencia de cuadro los padres deciden acudir a Urgencias donde es valorada por el Servicio de Psiquiatría y es diagnosticada de TOC con predominio de ideas obsesivas. Se pautó tratamiento con ser tralina (50 mg/día) y cloracepato dipotásico $(7,5$ $\mathrm{mg} /$ día). Dada la coincidencia temporal con la amigdalectomía se sugirió la posibilidad de que se tratara de un cuadro de PANDAS y se remitió para seguimiento al CSM.

Desde el CSM se contactó con el pediatra y éste añadió un tratamiento erradicador de estreptococo con amoxicilina a dosis de 40 $\mathrm{mg} / \mathrm{kg} /$ día cada 8 horas durante 21 días (la propuesta inicial de utilizar Penicilina Benzatina 1200000 U por pesar la niña más de $25 \mathrm{~kg}$ fue rechazada por la madre por el temor de la niña a los inyectables). Los síntomas del TOC remitieron en tres semanas. Posteriormente se fue disminuyendo la sertralina y el cloracepato de forma progresiva.

Tabla 1. Criterios diagnósticos de PANDAS

- Presencia de TOC y/o trastorno de tics.

- Inicio en la infancia (entre los 3 años y la pubertad)

- Curso episódico (inicio brusco de los síntomas seguido por una remisión gradual y posterior ausencia de síntomas hasta la reaparición brusca de los síntomas).

- Presencia de infección por estreptococo beta-hemolítico del grupo A (determinado por un cultivo faríngeo o elevación del título de anticuerpos antiestreptocócicos)

- Presencia de signos neurológicos anormales (movimientos anormales, hiperactividad...).

PANDAS: Pediatric Autoinmune Neuropsychiatric Disorders Associated with Streptococcal Infections. TOC: Trastorno obsesivo-compulsivo. 
A los dos meses del inicio del cuadro, coincidiendo con una infección faríngea, reapareció la misma sintomatología de forma brusca intensa. En esta ocasión no fue tratada con psicofármacos, sino exclusivamente con antibiótico (amoxicilina a la dosis habitual de 50 $\mathrm{mg} / \mathrm{Kg} /$ día) y el cuadro cedió en tres días. La detección de antiestreptolisinas, que había sido negativa antes de la amigdalectomía, fue en esta ocasión positiva.

La evolución posterior del cuadro ha sido favorable, con remisión sintomática total. Doce meses después del segundo episodio la paciente no ha vuelto a presentar infecciones respiratorias ni ha reaparecido la clínica obsesiva. En estos doce meses la paciente no ha necesitado psicofármacos.

\section{DISCUSIÓN}

La paciente presentada puede diagnosticarse de PANDAS ya que se trata de un TOC de inicio en la infancia, a una edad muy precoz, con inicio muy brusco, acompañado de clínica ansiosa e hiperactividad que en el primer episodio aparece tras la amigdalectomía y reaparece tras una infección faríngea, con positivización de las antiestreptolisinas-O (ASLO).

La etiopatogenia del TOC en estos cuadros se supone mediada por mecanismos autoinmunes, por autoanticuerpos que actuarían sobre los ganglios basales ${ }^{4}$ con un mecanismo similar al de la corea de Sydenham. Como marcador predictor del desarrollo de síntomas obsesivos y/o tics después de una infección estreptocócica se ha propuesto al antígeno linfocitario $\mathrm{B}$ D8/17. En los estudios realizados este antígeno aparece tanto en los afectos de corea de Sydenham como en los de PANDAS con mayor frecuencia que en los controles ${ }^{5,6}$ sugiriendo un mecanismo autoinmune similar en ambas patologías.

En nuestro caso, el hecho de que sea tras la amigdalectomía cuando aparecen los síntomas obsesivos nos hace pensar que la creación de autoanticuerpos tuvo lugar tras la intervención, lo que concuerda con la positivización de las antiestreptolisinas, que habían sido negativas antes a pesar de que la paciente sufriera amigadalitis de repetición.

Por otro lado, en la predisposición a desarrollar esta enfermedad tras una infección tiene importancia la carga familiar. La prevalencia de trastornos de tics y TOC en familiares de primer grado de los niños con PANDAS es superior a la de la población general y similar a la de los casos de tics o TOC no relacionados con infecciones por estreptococo ${ }^{7}$. En el caso descrito, la madre de la paciente tiene un TOC diagnosticado en la edad adulta.

Debe quedar claro que los casos de PANDAS son solamente un subgrupo de los TOC o trastornos de tics de aparición en la infancia, siendo la mayoría de los casos de estas patologías independientes de las infecciones estreptocócicas. Como estas infecciones son tan frecuentes en la infancia, es preciso tener una referencia longitudinal de la evolución para ver si hay relación entre la infección y la exacerbación de la clínica psiquiátrica, teniendo en cuenta que con frecuencia los síntomas agudos infecciosos ceden días antes de aparecer los obsesivos y/o tics. En cualquier caso, debe tenerse en cuenta que el diagnóstico de estos casos es básicamente clínico (infección-exacerbación psicopatológica) y que la solicitud de pruebas complementarias como la determinación de antiestreptolisinas, aunque puedan apoyarlo, no es decisiva dada su inespecificidad.

El tratamiento en los casos de PANDAS no está claramente definido. La mejoría del primer episodio del caso descrito podría ser atribuida tanto a los antibióticos como a los antidepresivos. Sin embargo el segundo episodio fue tratado sólo con antibióticos, lo que sugiere que la mejoría de ambos episodios fue debida al tratamiento antibiótico. Las investigaciones se están centrando en terapéuticas de tipo inmunológico (plasmaféresis ${ }^{8,9}$ y la administración de inmunoglobulinas ${ }^{9}$ ) y antibiótico (tratamiento antibiótico intensivo en el episodio centinela ${ }^{10} \mathrm{o}$ la profilaxis antibiótica ${ }^{11}$ similar a la realizada en la fiebre reumática). A pesar de la excelente respuesta de nuestra paciente al tratamiento antibiótico, no hay por el momento evidencias claras que indiquen que éste sea el tratamiento de elección.

El interés de esta paciente radica en que se trata, según por la bibliografía consultada, del primer caso de PANDAS iden- 
tificado en Navarra y también del primero descrito en la literatura en España. Además, se describe por vez primera la asociación entre PANDAS y amigdalectomía. Queremos destacar que a pesar de que se haya descrito el caso de dos gemelos, uno con un cuadro de TOC y otro con un trastorno de tics, que mejoraron tras haber sido amigdalectomizados ${ }^{12}$, en el caso aquí descrito ha sido precisamente la amigdalectomía el desencadenante del cuadro.

\section{BIBLIOGRAFÍA}

1. GRIMSHAW L. Obsessional disorders and neurological illness. J Neurol Neurosurg Psychiatry 1964; 27: 229-231.

2. Swedo SE, Rapoport JL, Cheslow DL, LeOnARD HL, Ayoub EM, Hosier DM et al. High prevalence of obsessive-compulsive symptoms in patients with Sydenham's chorea. Am J Psychiatry 1989; 146: 246-249.

3. Swedo SE, Leonard HL, Garvey M, Mittleman B, Allen AJ, Perlmutter $S$ et al. Pediatric autoimmune neuropsychiatric disorders associated with streptococcal infections: clinical description of the first 50 cases. Am J Psychiatry 1998;155: 264-271.

4. Giedd JN, Rapoport JL, Garvey MA, Perlmutter S, SwEDO SE. MRI assessment of children with obsessive-compulsive disorder or tics associated with streptococcal infection. Am J Psychiatry 2000; 157: 281-283.

5. MurPhy TK, Goodman WK, Fudge MW, Williams RC JR, AYouB EM, DALAL M et al. B lymphocyte antigen D8/17: a peripheral marker for childhood-onset obsessive-compulsive disorder and Tourette's syndrome? Am J Psychiatry 1997; 154: 402-407.

6. Swedo SE, Leonard HL, Mittleman BB, Allen AJ, RAPOPORT JL, Dow SP et al. Identification of children with pediatric autoimmune neuropsychiatric disorders associated with streptococcal infections by a marke associated with rheumatic fever. Am J Psychiatry 1997; 154: 110-112.

7. Lougee L, Perlmutter SJ, Nicolson R, Garvey MA, SwEDo SE. Psychiatric disorders in firstdegree relatives of children with pediatric autoimmune neuropsychiatric disorders associated with streptococcal infections (PANDAS). J Am Acad Child Adolesc Psychiatry 2000; 39: 1120-1126.

8. Nicolson R, Swedo SE, Lenane M, Bedwell J, WUDARSKY M, GOCHMAN P et al. An open trial of plasma exchange in childhood-onset obsessive-compulsive disorder without poststreptococcal exacerbations. J Am Acad Child Adolesc Psychiatry 2000; 39: 13131315.

9. Perlmutter SJ, Leitman SF, Garvey MA Hamburger S, Feldman E, LeONARd HL et al Therapeutic plasma exchange and intravenous immunoglobulin for obsessivecompulsive disorder and tic disorders in childhood. Lancet 1999; 354: 1153-1158.

10. Murphy ML, Pichichero ME. Prospective identification and treatment of children with pediatric autoimmune neuropsychiatric disorder associated with group A streptococcal infection (PANDAS). Arch Pediatr Adolesc Med 2002; 156: 356-361.

11. Garvey MA, Perlmutter SJ, Allen AJ, HAMburger S, LougeE L, LeONARD HL et al. A pilot study of penicillin prophylaxis for neuropsychiatric exacerbations triggered by streptococcal infections. Biol Psychiatry 1999; 45:1564-1571.

12. ORvidas LJ, Slattery MJ. Pediatric autoimmune neuropsychiatric disorders and streptococcal infections: role of otolaryngologist. Laryngoscope 2001; 111: 15151519. 